Communications in Physics, Vol.21, No. 3 (2011), pp. 245-252

\title{
INVESTIGATION OF THE EXAFS CUMULANTS OF SILICON AND GERMANIUM SEMICONDUCTORS BY STATISTICAL MOMENT METHOD: PRESSURE DEPENDENCE
}

\author{
HO KHAC HIEU \\ National University of Civil Engineering \\ and \\ Hanoi University of Science \\ VU VAN HUNG \\ Hanoi National University of Education \\ NGUYEN VAN HUNG \\ Hanoi University of Science
}

\begin{abstract}
Pressure dependence of Extended X-ray Absorption Fine Structure (EXAFS) cumulants of silicon and germanium have been investigated using the statistical moment method (SMM). Analytical expressions of the first and second cumulants of silicon and germanium have been derived. The equations of states for silicon and germanium semiconductors have been also obtained using which the pressure dependence of lattice constants and volume of these semiconductors have been estimated. Numerical results using the developed theories for these semiconductors are found to be in good and reasonable agreement with those of the other theories and with experiment.
\end{abstract}

\section{INTRODUCTION}

Two of the diamond-type semiconductors silicon and germanium play an important role in technological and especially in electronic applications. The understanding of thermodynamic properties of these semiconductors is very useful. One of the most effective methods for investigation of structure and thermodynamic properties of crystals is EXAFS [1]. The anharmonic EXAFS providing information on structure and thermodynamic parameters of substances has been analyzed by means of cumulant expansion approach $[1,2]$. In this formulation, an EXAFS oscillation function $\chi(k)$ is given by [3]

$$
\chi(k)=\frac{F(k)}{k R^{2}} e^{-2 R / \lambda(k)} \operatorname{Im}\left\{e^{i \phi(k)} \exp \left[2 i k R+\sum_{n} \frac{(2 i k)^{n}}{n !} \sigma^{(n)}\right]\right\},
$$

where $k$ and $\lambda$ are the wave number and mean free path of emitted photoelectrons, $F(k)$ is the real atomic backscattering amplitude, $\phi(k)$ is the net phase shift, and $\sigma^{(n)}(n=1$, $2,3, \ldots)$ are the cumulants.

The pressure dependence of the EXAFS second cumulant has been measured at the Stanford Synchrotron Radiation Laboratory (SSRL, USA) for $\mathrm{Cu}$ [6], and at the Laboratoire Pour I'Utisation du Rayonnement Electromagnétique (LURE) (Orsay, France) 
for $\operatorname{Kr}[7,8]$. Such pressure effects have been calculated by correlated Debye model [6], as well as by Monte-Carlo (MC) simulation [7] and by Loubeyre's model [8] to interpret experimental results.

Some EXAFS studies on crystalline and amorphous Ge under pressure have already been presented by Kawamura et al. [9] and Freund et al. [10]. The EXAFS spectra of Ge near K-edge in diamond-type Ge under high temperature and high pressure were measured using a cubic-anvil-type apparatus (MAX90) with synchrotron radiation from the Photon Factory, Tsukuba, Japan [11]. Theoretical approach has been done to estimate the second cumulant on the basis of the isothermal equation of state of Ge up to the pressure of 10.6 GPa [11].

EXAFS is sensitive to pressure $[4,5]$ which can cause certain changes of cumulants leading to uncertainties in physical information taken from EXAFS. Therefore, the investigation of pressure effects of cumulants becomes very useful.

Recently, the statistical moment method (SMM) has been used for calculation of temperature dependence of EXAFS cumulants of silicon and germanium crystals at zero pressure [12]. The purpose of this work is to develop the SMM for calculating and analyzing the pressure dependence of cumulants of silicon and germanium crystals at a given temperature. The equation of state has been also obtained to determine pressure dependence of lattice constants and volumes of silicon and germanium crystals. The calculated results using our derived theory are compared to experiment and to those of the other theories $[11,19,20-29]$ showing a good and reasonable agreement.

\section{FORMALISM}

\section{II.1. General Formula of EXAFS Cumulants}

Firstly, we present the SMM for calculating the cumulants of silicon and germanium semiconductors by using the Stillinger-Weber potentials which consist of two-body and three-body terms

$$
\varphi_{i}=\sum_{j} \Phi_{i j}\left(r_{i}, r_{j}\right)+\sum_{j, k} W_{i j k}\left(r_{i}, r_{j}, r_{k}\right)
$$

where

$$
\Phi_{i j}\left(r_{i}, r_{j}\right)= \begin{cases}\varepsilon A\left[B\left(\frac{r_{i j}}{\sigma}\right)^{-4}-1\right] \exp \left[\left(\frac{r_{i j}}{\sigma}-b\right)^{-1}\right], & \frac{r_{i j}}{\sigma}<b \\ 0, & \frac{r_{i j}}{\sigma} \geq b\end{cases}
$$

and

$$
W_{i j k}\left(r_{i}, r_{j}, r_{k}\right)=\varepsilon \lambda \exp \left[\gamma\left(\frac{r_{i j}}{\sigma}-b\right)^{-1}+\gamma\left(\frac{r_{i k}}{\sigma}-b\right)^{-1}\right]\left(\cos \theta_{i j k}+\frac{1}{3}\right)^{2} .
$$

where $\theta_{i j k}$ is the angle between bond $i j$ and bond $i k$.

The effective interatomic potentials of the system is given by

$$
U=\sum_{i} \varphi_{i}=\frac{1}{2} \sum_{i, j} \Phi_{i j}\left(r_{i}, r_{j}\right)+\frac{1}{3} \sum_{i, j, k} W_{i j k}\left(r_{i}, r_{j}, r_{k}\right)
$$

where $\varphi_{i}$ is the internal energy associated with atom $i$. 
Using the SMM [13], one can get power moments of the atomic displacement of diamond-type semiconductor $y_{0}(T)$, taking into account the anharmonic effects of the thermal lattice vibrations

$$
y_{0}=y_{0}^{\prime}-\frac{\beta}{3 \gamma}+\frac{1}{K}\left(1+\frac{6 \gamma^{2} \theta^{2}}{K^{4}}\right)\left[\frac{1}{3}-\frac{2 \gamma \theta}{3 k^{2}}(x \operatorname{coth} x-1)-\frac{2 \beta^{2}}{27 \gamma k}\right] \frac{\beta k}{\gamma},
$$

where

$$
\begin{gathered}
k=\frac{1}{2} \sum_{i}\left(\frac{\partial^{2} \varphi_{i}}{\partial u_{i x}^{2}}\right)_{e q} \equiv m \omega^{2} ; \gamma=\frac{1}{12}\left\{\sum_{i}\left[\left(\frac{\partial^{4} \varphi_{i}}{\partial u_{i x}^{4}}\right)_{e q}+6\left(\frac{\partial^{4} \varphi_{i}}{\partial u_{i x}^{2} \partial u_{i y}^{2}}\right)_{e q}\right]\right\} ; \\
\beta=\left(\frac{\partial^{3} \varphi_{i}}{\partial u_{i x} \partial u_{i y} \partial u_{i z}}\right)_{e q} ; \quad y_{0}^{\prime} \approx \sqrt{\frac{2 \gamma \theta^{2}}{3 K^{3}}} A ; \theta=k_{B} T ; x=\hbar \omega / 2 \theta \\
K=k-\frac{\beta^{2}}{3 \gamma} ; A=a_{1}+\frac{\gamma^{2} \theta^{2}}{K^{4}} a_{2}+\frac{\gamma^{3} \theta^{3}}{K^{6}} a_{3}+\frac{\gamma^{4} \theta^{4}}{K^{8}} a_{4}+\frac{\gamma^{5} \theta^{5}}{K^{10}} a_{5}+\frac{\gamma^{6} \theta^{6}}{K^{12}} a_{6},
\end{gathered}
$$

and $k_{B}$ is Boltzmann constant.

The average nearest-neighbor distance (NND) of atoms in crystal at a given temperature $T$ can be determined as

$$
r(T)=r(0)+y_{0}(T),
$$

where $r(0)$ denotes the NND $r(T)$ at the temperature $0 K$, which can be determined from experiment or from the minimum condition of the potential energy of the crystal. The lattice constant $a_{h}$ of the diamond-type semiconductor can be calculated easily using the relation $a_{h}=r(T) 4 / \sqrt{3}$.

Using $x=r-r_{0}$ as the deviation of instantaneous bond length $r$ from its equilibrium value $r_{0}$, we derive the first order cumulant:

$$
\begin{aligned}
\sigma^{(1)} & =\langle x\rangle=\left\langle r-r_{0}\right\rangle \approx r(T)-r(0)=y_{0}(T) \\
& =\sqrt{\frac{2 \gamma \theta^{2}}{3 K^{3}} A}-\frac{\beta}{3 \gamma}+\frac{1}{K}\left(1+\frac{6 \gamma^{2} \theta^{2}}{K^{4}}\right)\left[\frac{1}{3}-\frac{2 \gamma \theta}{3 k^{2}}(x \operatorname{coth} x-1)-\frac{2 \beta^{2}}{27 \gamma k}\right] \frac{\beta k}{\gamma} .
\end{aligned}
$$

The second cumulant $\sigma^{(2)}=\sigma^{2}$ is an important factor in EXAFS analysis since the thermal lattice vibrations influence sensitively the XAFS amplitudes through the Debye-Waller factor $e^{-W} \sim \exp \left(-2 \sigma^{2} k^{2}\right)$. The parallel mean square relative displacement (MSRD) to a good approximation corresponds to the second cumulant

$$
\sigma^{2}=\left\langle\left[\vec{R} .\left(\vec{u}_{i}-\vec{u}_{0}\right)\right]^{2}\right\rangle=\left\langle u_{i}^{2}\right\rangle+\left\langle u_{0}^{2}\right\rangle-2\left\langle u_{i} u_{0}\right\rangle
$$

Using the expression of the second order moment $[12,13]$, we obtain the mean-square displacement (MSD)

$$
\left\langle u_{i}^{2}\right\rangle=\left\langle u_{i}\right\rangle^{2}+\theta A_{1}+\frac{\theta}{k}(x \operatorname{coth} x-1),
$$

where

$$
A_{1}=\frac{1}{K}\left[1+\frac{2 \gamma^{2} \theta^{2}}{K^{4}}\left(1+\frac{x \operatorname{coth} x}{2}\right)(x \operatorname{coth} x+1)\right] \text {. }
$$


For crystals that have a basic cubic structure, such as fcc, any directional dependence of $\left\langle u^{2}\right\rangle$ must have cubic symmetry. The quadratic contribution to the Debye-Waller factor is necessarily isotropic. For crystals with a basic hexagonal structure, such as hcp, $\left\langle u^{2}\right\rangle$ is not isotropic; in general, the components along the $a$ and $c$ axes, $\left\langle u_{a}^{2}\right\rangle$ and $\left\langle u_{c}^{2}\right\rangle$, for hcp crystals, are not equal. Hence

$$
\left\langle u_{j}^{2}\right\rangle \approx\left\langle u_{0}^{2}\right\rangle \quad ; \quad\left\langle u_{j} u_{0}\right\rangle \approx\left\langle u_{j}\right\rangle\left\langle u_{0}\right\rangle .
$$

Therefore, from Eqs. (12), (13), and (15), we derived the second cumulant expression of the diamond-type semiconductor as

$$
\sigma^{2}(T) \approx \frac{4 \gamma^{2} \theta^{3}}{K^{5}}\left(1+\frac{x \operatorname{coth} x}{2}\right)(x \operatorname{coth} x+1)+\frac{2 \theta}{k} x \operatorname{coth} x+2 \theta\left(\frac{1}{K}-\frac{1}{k}\right) .
$$

\section{II.2. Equation of state and pressure dependence of EXAFS cumulants}

From the expression for the Helmholtz free energy of system [14-16], the pressure $P$ of the diamond-type semiconductors can be written in the form

$$
P=-\left(\frac{\partial \psi}{\partial V}\right)_{T}=-\frac{r}{3 v} \frac{\partial \varphi_{0}}{\partial r}+\frac{3 \gamma_{G} \theta}{v}
$$

where $\gamma_{G}$ is the Grüneisen constant, $v$ is the atomic volume..

From the Eq. (17) one can find the NND $r(P, T)$ at pressure $P$ and temperature $T$. However, for numerical calculations, it is convenient to determine firstly the NND of crystals $r(P, 0)$ at pressure $P$ and at absolute zero temperature $T=0 K$. For $T=0 K$ temperature, Eq. (17) is reduced to

$$
P v=-a\left[\frac{1}{3} \frac{\partial \varphi_{0}}{\partial r}+\frac{\hbar \omega}{4 k} \frac{\partial k}{\partial r}\right] .
$$

Eq. (18) can be solved using a computational program to find out the values of the NND $r(P, 0)$ of the semiconductors. From the obtained results of NND $r(P, 0)$ one can find the values of parameters $K(P, 0), k(P, 0), \gamma(P, 0)$ and $\beta(P, 0)$ at pressure $P$ and temperature $T=0 K$. Then, we can find $r(P, T)$ at pressure $P$ and temperature $T$ as

$$
r(P, T)=r(P, 0)+y_{0}(P, T),
$$

where $y_{0}(P, T)$ is the displacement of an atom from the equilibrium position at pressure $P$ and temperature $T$. This quantity can be determined by substituting the values of $K(P, 0), k(P, 0), \gamma(P, 0)$ and $\beta(P, 0)$ into Eq. (6).

Using the above formula of NND $r(P, T)$, we can find the change of the crystal volume under pressure $P$ at a given temperature $T$ as

$$
\frac{V}{V_{0}}=\frac{r^{3}(P, T)}{r^{3}(0, T)} .
$$

The pressure dependence of MSD of crystals can be obtained as

$$
\left\langle u_{i}^{2}\right\rangle(P, T)=y_{0}^{2}(P, T)+\theta A_{1}(P, T)+\frac{\theta}{k(P, 0)}(x \operatorname{coth} x-1),
$$


where

$$
A_{1}(P, T)=\frac{1}{K(P, 0)}\left[1+\frac{2 \gamma^{2}(P, 0) \theta^{2}}{K^{4}(P, 0)}\left(1+\frac{x \operatorname{coth} x}{2}\right)(x \operatorname{coth} x+1)\right] .
$$

Substituting the values of $K(P, 0), k(P, 0), \gamma(P, 0)$ and $\beta(P, 0)$ into Eqs. (11,16), we can find out the values of the first, second cumulants of silicon and germanium semiconductors under pressure $P$ at the given temperature $T$.

\section{NUMERICAL RESULTS AND DISCUSSIONS}

Now we apply the expressions derived in previous section to determine the pressure dependence of lattice constant, the change of volume, the first and second cumulants of silicon and germanium semiconductors. The interaction potential between the two intermediate atoms used in this article is the Stillinger-Weber potential, where the potential parameters of Ge and Si semiconductors are shown in Table $1(b=1.2, \gamma=1.8)[17,18]$.

Table 1. The Stillinger-Weber potential parameters [17, 18]

\begin{tabular}{|l|c|c|c|l|l|}
\hline \hline Potential parameters & $\epsilon(\mathrm{eV})$ & $\mathrm{A}$ & $\mathrm{B}$ & $\sigma(\AA)$ & $\lambda$ \\
\hline \hline $\mathrm{Si}$ & 2.17 & 7.049556277 & 0.6022245584 & 2.0951 & 21.0 \\
\hline $\mathrm{Ge}$ & 1.93 & 7.049556277 & 0.6022245584 & 2.181 & 31.0 \\
\hline \hline
\end{tabular}

In Fig. 1a we show the pressure dependence of NND of Ge crystal at room temperature. The pressure dependence of the calculated NND is consistent with the one of A. Yoshiasa et al.'s results [11]. The lattice constant of Ge crystal can be calculated using the values of NND. The change of volume under pressure up to $11 \mathrm{GPa}$ of Ge crystal is showed in Fig. 1b. Our calculation results have been compared to available experimental data [20] as well as to the other theoretical results [11, 19] showing a good agreement.
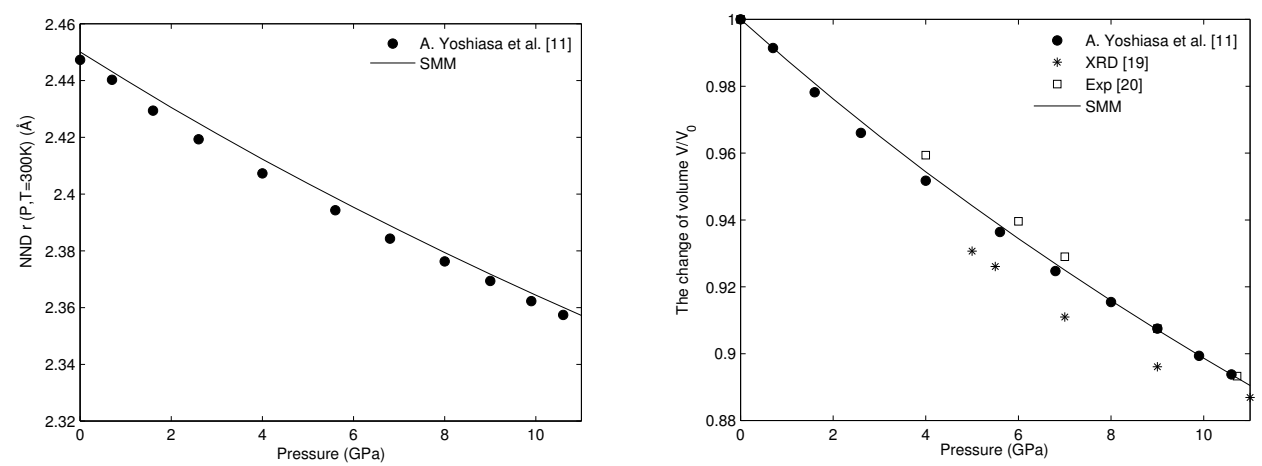

Fig. 1. (a) Pressure dependence of NND of Ge; (b) Pressure dependence of volume of Ge. 

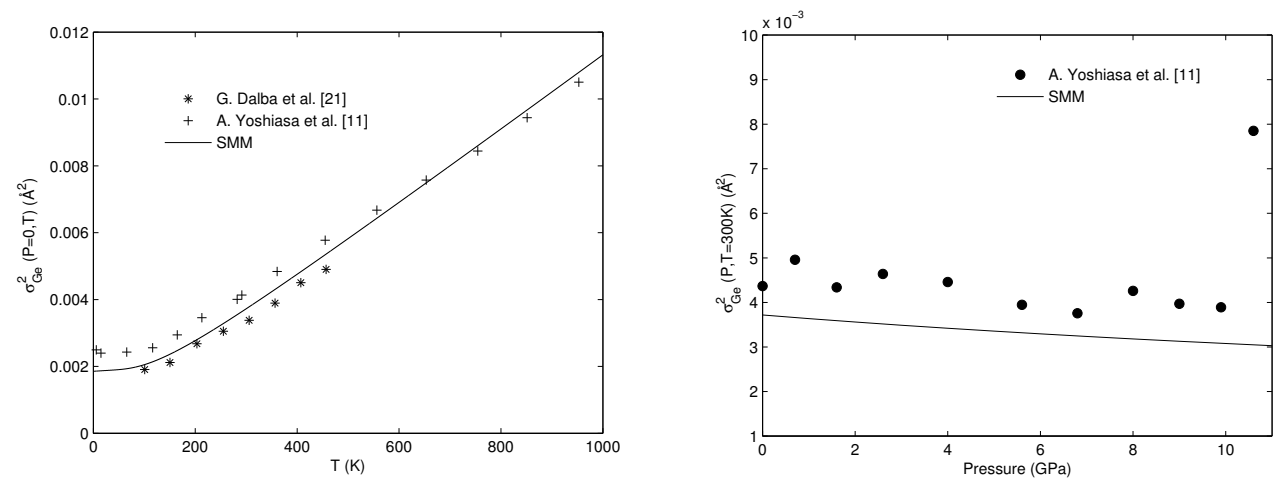

Fig. 2. (a) Temperature dependence of DWF of Ge; (b) Pressure dependence of DWF of Ge.

Fig. 2a. shows the temperature dependence of second cumulant or Debye-Waller factor of germanium crystal at zero pressure. Our calculated results of second cumulant have been compared to the values of A. Yoshiasa et al. [11] and G. Dalba et al. [21]. They are found to be in good agreement with those of G. Dalba et al. [21] and in a reasonable agreement with the results of A. Yoshiasa et al. [11]. In higher pressure, the calculated pressure dependence of Debye-Waller factors at temperature $T=300 \mathrm{~K}$ does not agree well with A. Yoshiasa et al.'s values (Fig. 2b). However, the decreasing ratio between our calculated results and A. Yoshiasa et al.'s values is similar. It denotes that, the SMM is still good for calculating the relative change of the Debye-Waller factor of Ge crystal under pressure.

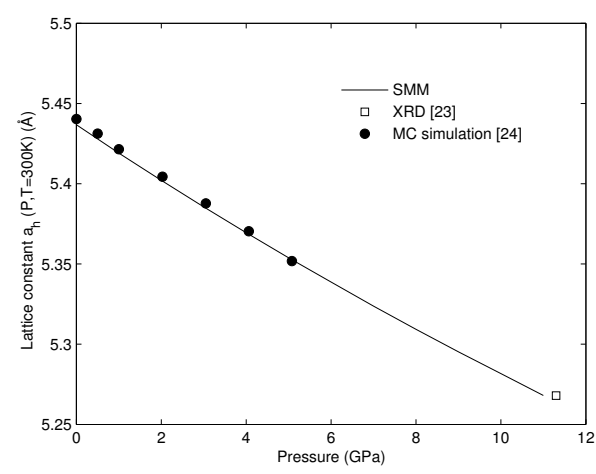

(a)

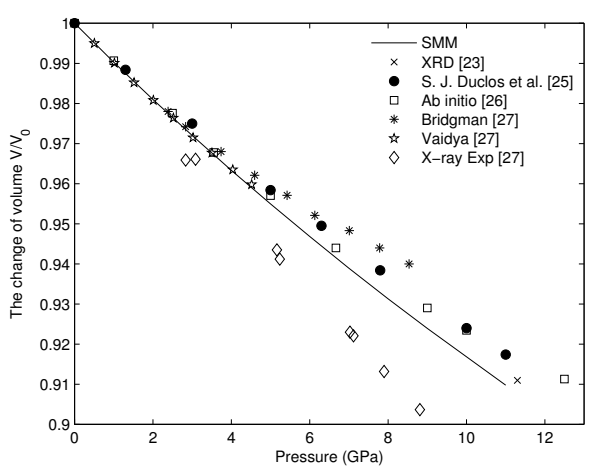

(b)

Fig. 3. (a) Pressure dependent lattice constants of Si; (b) Pressure dependence of volume of Si.

In Fig. 3a, we plot the pressure dependence of lattice constant of silicon crystal calculated by SMM as well as the values of XRD [23] and Monte-Carlo simulations [24]. 
The pressure-volume relations of $\mathrm{Si}$ semiconductor have been showed in Fig. 3b. Our calculated $V / V_{0}$ are compared to experiment $[23,27]$ and to other theoretical results [2527 showing the good agreement.

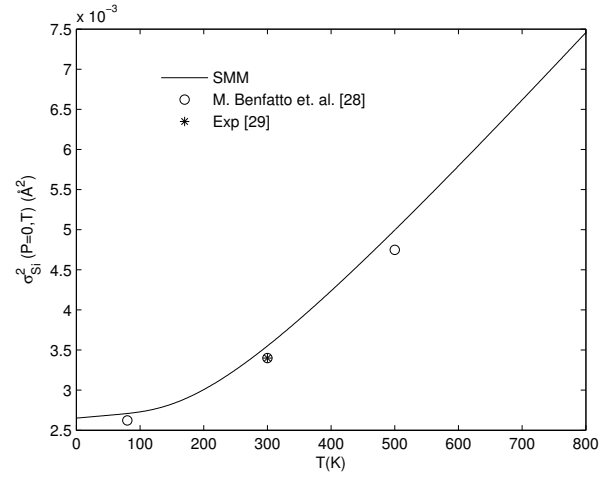

(a)

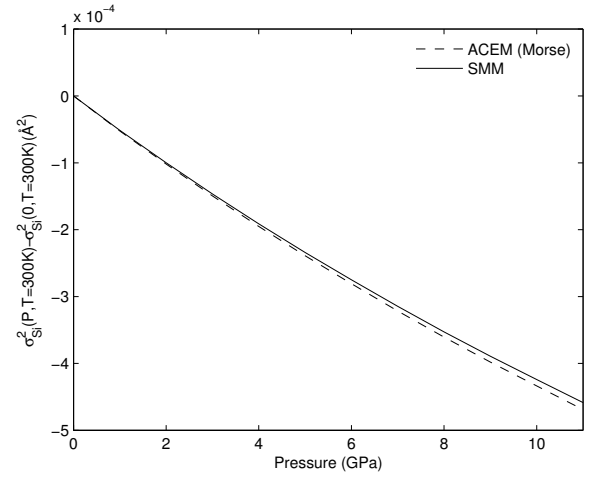

(b)

Fig. 4. (a) Temperature dependence of DWF of Si; (b) Pressure dependence of DWF of Si.

Our calculated results for the temperature dependence of Debye-Waller factor of Si crystal at zero pressure has been showed in Fig.4a. They agree with the available experimental data [29] and with M. Benfatto et al.'s calculated results [28].

Fig. $4 \mathrm{~b}$ shows the pressure dependence of the change of second cumulant of $\mathrm{Si}$ crystal. Because of the lack of experimental data as well as other theoretical calculations, we compared the results calculated by our SMM with those calculated by the anharmonic correlated Einstein model (ACEM) [30] using Morse potential. This figure shows the agreement between results of two methods.

\section{CONCLUSIONS}

In this work, the pressure effects in thermodynamic quantities of diamond-type silicon and germanium semiconductors have been investigated by using the SMM which has been applied to three-dimensional crystals. Moreover, the present SMM formalism takes into account the quantum-mechanical zero-point vibrations as well as the higherorder anharmonic terms in the atomic displacements.

Our development is establishing and solving equation of state to get the pressure dependence of the lattice bond length, and then is the derivation of the analytical expressions of pressure dependence for the first and second EXAFS cumulants, the change of volume of diamond-type semiconductors.

The good and reasonable agreement of our calculated results with experiment and with those of the other theories denotes the efficiency of our derived theory in the investigation of the pressure dependence of thermodynamic quantities of semiconductors. 


\section{ACKNOWLEDGEMENTS}

This work is supported by the research project No. 103.01.09.09 of NAFOSTED. One of the authors (V. V. H.) acknowledges the partial support of the research project No. 103.01.2609 of NAFOSTED.

\section{REFERENCES}

[1] E. D. Crozier, J. J. Rehr, and R. Ingalls, in X-ray Absorption, edited by D. C. Koningsberger and R. Prins (Wiley, New York, 1988) chapter 9.

[2] G. Bunker, Nucl. Instr. Meth. Phys. Res. 207 (1983) 437-444.

[3] N. V. Hung, N. B. Duc, and R. R. Frahm, J. Phys. Soc. Jpn. 72 (2003) 1254.

[4] R. Ingalls, G. A. Garcia, and E. A. Stern, Phys. Rev. Lett. 40 (1978) 334.

[5] R. Ingalls, E. D. Crozier, J. E. Whitemore, A. J. Seary, and J. M. Tranquada, J. Appl. Phys. 51 (1980) 3158.

[6] J. Freund, R. Ingalls and E. D. Crozier, Phys. Rev. B39 (1989) 12537.

[7] A. Di Cicco, A. Filipponi, J. P. Itie, and A. Polian, Phys. Rev. B54 (1996) 9086 .

[8] A. Polian, J. P. Itie, E. Daartyge, A. Fontaine, and G. Tourillon, Phys. Rev. B39 (1989) 3369.

[9] T. Kawamura, O. Shimomura, T. Fukamachi, and P. H. Fuoss, Acta Cryst. A37 (1981) 653-658.

[10] J. Freund, R. Ingalls, R. and E. D. Crozier, J. Phys. Chem. 94 (1990) 1087-1090.

[11] A. Yoshiasa, T. Nagai, O. Ohtaka, O. Kamishima, and O. Shimomur, J. Synchrotron Rad. 6 (1999) 43-49.

[12] V. V. Hung, H. K. Hieu, and K. Masuda-Jindo, Comput. Mater. Sci. 49 (2010), pp S214-S217.

[13] V. V. Hung, K. Masuda-Jindo, and P. T. M. Hanh, J. Phys. Condens. Matter. 18 (2006) 283-293.

[14] N. Tang, and V. V. Hung, Phys. Stat. Sol. (b) 149 (1988), 511; 161 (1990) 165.

[15] K. Masuda-Jindo, V. V. Hung, and P. D. Tam, Phys. Rev. B67 (2003) 094301.

[16] K. Masuda-Jindo, S. R. Nishitani, and V. V. Hung, Phys. Rev. B70 (2004) 184122.

[17] S. Erkoc, Phys. Reports,278 (2) (1997) 81-88.

[18] F. H. Stillinger and T. A. Weber, Phys. Rev. B31 (1985) 5262.

[19] G. A. Voronin, C. Pantea, T. W. Zerda, J. Zhang, L. Wang, and Y. Zhao, J. Phys. Chem. Sol. 64 (2003) 2113-2119

[20] C. S. Menoni, J. Z. Hu, and placeI. L. Spain, Phys. Rev. B34 (1986) 362.

[21] G. Dalba, P. Fornasini, D. Diop, M. Grazioli, and F. Rocca, J. Non-Crys. Sol. 164 (1993) 159-162.

[22] M. Senoo, H. Mii, and I. Fujishiro, J .Phys. Soc. Jpn. 41 (5) (1976) 1562-1569.

[23] J. Z. Hu, L. D. Merkle, C. S. Menoni, and I. L. Spain, Phys. Rev. B34 (1986) 4679.

[24] J. C. Noya, C. P. Herrero, and R. Ramirez, Phys. Rev. B53 (1996) 9869.

[25] S. J. Duclos, Y. K. Vohra, and A. L. Ruoff, Phys. Rev. B41 (1990) 12021.

[26] M. Durandurdu, D. A. Drabold, Phys. Rev. B67 (2003) 212101.

[27] M. Senoo, H. Mii and placeI. Fujishiro, J. Phys. Soc. Jpn. 41 (1976) 1562.

[28] M. Benfatto, C. R. Natoli, and A. Filipponi, Phys. Rev. B40 (1989) 9626.

[29] A. Filipponi, F. Evangelisti, M. Benfatto, S. Mobilio, and C. R. Natoli, Phys. Rev. B40 (1989) 9636.

[30] V. V. Hung, H. K. Hieu and N. V. Hung, Comm. Phys. 20 (2010) 219-225.

Received 15 September 2010. 